\title{
MAŁGORZATA SOKOŁOWICZ
}

Université de Varsovie

\section{Le voyage en Orient de Gustave Flaubert : révolution ou évolutiondans l'approche de}

l'Orient?

A u salon de 1834, Eugène Delacroix expose une

1 grande toile mettant en scène trois jeunes femmes, bras et jambes dénudés, sensualité et attitude relâchées : Les femmes d'Alger dans leur appartement. Pour les critiques et le public, c'est un choc. Même Frédéric Chopin, ami du peintre, reste interloqué. On parle d'une révolution. Pourquoi ? Le réalisme du tableau est très embarrassant pour un public habitué à un Orient fantasmé : les tenues, les bijoux sont bien authentiques, les postures des femmes relèvent d'un naturalisme cru. Avant, les peintres (Delacroix y compris) avaient tendance à truquer leur Orient ${ }^{1}$, d'autant plus que ce monde dit oriental se prêtait facilement au truquage : "On ne s'attend pas à ce que nous donnions ici les délimitations géographiques de ce pays indéterminé qu'on appelle I'“Orient" ", lit-on dans le Dictionnaire Larousse du XIXe siècle ; "Rien de plus vague, rien de plus mal défini que la

\footnotetext{
${ }^{1}$ Cf. M.-C. Genet-Delacroix, "Delacroix et les "Néos" : pour le vrai contre le faux ", [dans :] Sociétés \& Représentations, 2005, n० 20, p. 242 ; P. Vauday, La Décolonisation du tableau. Art et poétique au XIXe siècle. Delacroix, Gauguin, Paris, Seuil, 2006, p. 13-14 et le 9e épisode de la série Les petits secrets des grands tableaux : C. Cogitore, "Femmes d'Alger dans leur appartement ", 1834, Eugène Delacroix, ARTE France, 2015. Le tableau Femmes d'Alger dans leur appartement, huile sur toile, $180 \times 229$ $\mathrm{cm}$ se trouve actuellement dans le Musée du Louvre et s'affiche sur le site du musée : http://www.louvre.fr/oeuvre-notices/femmes-dalger-dans-leurappartement.
} 
contrée à laquelle on applique ce nom ${ }^{2}{ }^{2}$. L'Orient du XIXe siècle était tissé de rêves et la beauté du rêve réside dans son caractère flou, indéfini et imaginaire. Par son aspect réaliste, naturaliste même $^{3}$, la toile de Delacroix éveillait un malaise, une gêne. C'était une vision trop palpable, trop concrète, trop solide, au demeurant trop réelle pour ceux qui s'étaient habitués au rêve.

En 1849, Gustave Flaubert et son ami photographe et journaliste, Maxime Du Camp, partent en Orient. Leur voyage est, selon Jean-Claude Berchet, "le modèle du "périple" idéal " de l'époque. Les deux jeunes hommes visitent l'Égypte, la Palestine, la Syrie, le Liban, l'Asie Mineure, Constantinople, Athènes et la Grèce ${ }^{4}$. Durant l'expédition, Flaubert écrit des lettres et note ses impressions de voyage. Entre 1850 et 1851, il rédige ce qu'on appelle le Voyage en Égypte, texte publié en 1910 par la nièce de l'écrivain, Caroline Franklin-Grout, dans une version épurée. Le manuscrit n'est retrouvé qu'en 1989 : un collectionneur anonyme l'achète aux enchères et permet à Pierre-Marc de Biasi d'en profiter et de publier le texte non-censurés. C'est ainsi que la version originale des notes voit le jour, texte qui est en opposition absolue avec les récits de voyage, ou même les notes de voyage, de l'époque. On se rappelle le début des Femmes du Caire du Voyage en Orient de Gérard de Nerval où le narrateur regarde des femmes voilées dans la rue ${ }^{6}$. "C'est bien là le

\footnotetext{
2 P. Larousse, "Orient ", [dans :] Grand dictionnaire universel du XIXe siècle, Paris, Larousse, 1874, t. 11, s. 1463.

${ }^{3}$ Cf. M.-C. Genet-Delacroix, "Delacroix et les "Néos" : pour le vrai contre le faux », op. cit., p. 242.

${ }^{4}$ Cf. J.-C. Berchet, "Introduction ", [dans :] Le voyage en Orient. Anthologie des voyageurs français dans le Levant au XIXe siècle, J.-C. Berchet (éd. critique), Paris, Robert Laffont, 1985, p. 10.

${ }^{5}$ Cf. E. Sturm, "Voyage en Égypte by Gustave Flaubert and Pierre-Marc de Biasi », [dans :] The French Review, 1993, n 2 (67), p. 359-360.

${ }^{6}$ Nerval part en voyage oriental en 1843, seulement six ans avant Flaubert, c'est pourquoi il nous servira ici de point de référence privilégié. Le voyage en Orient (ses buts, itinéraires et particularités) change avec le temps et s'attache profondément au moment historique et à la généra-
} 
pays des rêves et de l'illusion ", s'enthousiasme-t-il, en précisant que, malgré le voile, "l'on peut toujours entrevoir quelque chose de ce qui est forme, grâce, jeunesse et beauté "». Et Flaubert ? Flaubert décrit un coït : "Nous nous sommes couchés ensemble sur la natte - chairs dures et fraîches - des fesses de bronze - les grandes lèvres coupées, le poil rasé. L'impression de son con était celle d'une graisse sèche ${ }^{8}$. L'auteur de Madame Bovary découvre l'Orient avec ses sens : mange, fume, se fait laver et masser au hammam, se paie des danseuses et des prostituées. II veut tout voir et tout faire, spécialement ce qu'il n'aurait jamais pu éprouver en Occident ${ }^{9}$. Et il décrit tout cela pour retrouver ensuite la même sensation ${ }^{10}$.

Flaubert cherche en Orient de " bizarres jouissances $»^{11}$ qui ne sont pas du tout imaginaires, mais bien palpables et

tion concernés. Voilà son caractère évolutif par excellence. $C f$. C. Berchet, "Introduction », op. cit., p. 3-20, mais aussi par exemple S. Moussa, "Signatures : ombre et lumière de l'écrivain dans la Correspondance d'Orient de Flaubert ", [dans :] Littérature, 1996, n 104, p. 76-77 ou F. Lacoste, "L'Orient de Flaubert ", [dans :] Romantisme, 2003, n 119, p. 73.

${ }^{7}$ G. de Nerval, Le Voyage en Orient, M. Jeanneret (éd. critique), Paris, Garnier-Flammarion, 1980, t. 1, p. 151.

8 G. Flaubert, Voyage en Orient, C. Gothot-Mersch (éd. critique), Paris, Gallimard, 2006, p. 88. Les citations suivantes venant de cette édition seront marquées par les initiales du titre - VO - et le numéro de page indiqués entre parenthèses dans le texte principal.

${ }^{9}$ Cf. A. Buisine, L'Orient voilé, Paris, Zulma, 1993, p. 108. Thierry Poyet parle même d'une " libération morale et psychique " absolue et de " la possibilité de laisser s'exprimer enfin, plus librement, une sexualité puissante " ( $C f$. Th. Poyet, "Le voyeur et sa sexualité. Flaubert ou l'interdit des sens levé pour rien ", [dans :] Ph. Antoine (dir.), Sur les pas de Flaubert. Approches sensibles du paysage, Amsterdam, Rodopi, 2014, p. 169-170).

10 II l'explique dans une lettre à Louise Colet : " Tu aurais voulu que ton nom revînt plus souvent sous ma plume. Mais remarque que je n'ai pas écrit une seule réflexion. Je formulais seulement de la façon la plus courte l'indispensable, c'est-à-dire la sensation, et non le rêve, ni la pensée » (G. Flaubert, lettre à Louise Colet du 27 mars 1853, [dans :] Correspondance. II (juillet 1851 - décembre 1858), J. Bruneau (éd. critique), Paris, Gallimard, 1980, p. 281).

${ }^{11}$ C'est l'expression qu'Edward Saïd emprunte à la Description de l'Égypte. 
réelles. Est-ce une révolution dans l'approche de l'Orient au XIXe siècle ou plutôt une évolution, conséquence naturelle du désir de réaliser ses rêves ? Telle est la question à laquelle nous essayerons de répondre dans notre article. Nous voudrions le faire en trois mouvements. Premièrement, nous parlerons brièvement de l'histoire de l'Orient rêvé, des origines de l'amour pour les odalisques picturales et littéraires. Ensuite, nous nous concentrerons sur quelques voyages en Orient faits dans les années 1840 et la rencontre du rêve et de la réalité décrite par d'autres voyageurs. Pour finir, nous analyserons la sexualisation de l'expérience orientale, tellement visible dans l'œuvre de Flaubert.

\section{L'Orient rêvé, l'Orient désiré}

La citation venant de Rage et impuissance, texte rédigé par Flaubert à l'âge de 14 ans, est devenue célèbre parmi les spécialistes en orientalisme. Le personnage principal, M. Ohmlin, est enterré vivant et fait un songe :

II rêvait l'Orient, [...] l'Orient avec sa poésie toute d'amour et d'encens [...], l'Orient avec ses sérails, séjour de fraîches voluptés. [...] il rêvait des lèvres de femmes pures et rosées, il rêvait de grands yeux noirs qui n'avaient d'amour que pour lui, il rêvait cette peau brune et olivâtre des femmes de l'Asie, doux satin qu'effleure si souvent dans ses nuits le poète qui les rêve $[\ldots] .^{12}$

Voilà l'Orient rêvé par excellence : sensuel, luxueux et luxurieux, avec sa figure centrale, la belle femme au corps agile. Quand l'Orient devient-il cette contrée du rêve et du désir ? Dans sa fameuse anthologie, Le voyage en Orient, Jean-Claude Berchet parle de la prolifération des voyages des Européens dans " les pays riverains de la Méditerranée orientale » à la fin de la Renaissance et puis tout au

Cf. E. Saïd, L'Orientalisme. L'Orient créé par l'Occident, trad. C. Malamoud, Paris, Éditions du Seuil, 2005, p. 223.

12 G. Flaubert, Rage et impuissance, [dans :] Mémoires d'un fou, Novembre et autres textes de jeunesse, Y. Leclerc (éd. critique), Paris, Flammarion, 1991, p. 130. 
long du XVIIe siècle et commente : " C'est pourquoi, on le soutiendra sans paradoxe, lorsque Galland publie sa fameuse traduction des Mille et Une Nuits (1704-1717), il inaugure bien moins qu'il ne termine, dans la splendeur du bouquet final, un grand siècle orientaliste ${ }^{13}$.

Et pourtant, Les Mille et une nuits servent de point de référence : les infidélités des femmes orientales très sévèrement punies par leurs maris contribuent à l'émergence de l'image romantique d'un Orient cruel et érotique à la fois dont parle Mario Praz ${ }^{14}$. La sultane mélancolique du Clair de lune hugolien n'entend-elle pas d'étranges bruits sortant de sacs lourds jetés dans le Bosphore ? Ce sont des sanglots de femmes punies ainsi de leur infidélité réelle ou présuméé ${ }^{15}$; la procédure est d'ailleurs décrite par Byron dans son Giaour. La belle Juive de La Sultane favorite ne réclame-t-elle pas au sultan la mort de ses rivales ${ }^{16}$ ? Éros et Thanatos s'unissent dans le rêve oriental.

Et puis, il y a des voyageurs. Lady Mary Montagu suit son mari, ambassadeur à Constantinople, découvre le monde féminin de l'Empire Ottoman et le décrit dans ses lettres, publiées en 1762. C'est avant tout la description du bain turc qui peut éveiller l'imagination d'un Européen :

Il est difficile de se représenter le charme d'un pareil spectacle. La plupart de ces jeunes filles étaient parfaitement bien faites et d'une blancheur

13 J.-C. Berchet, "Introduction », op. cit., p. 3.

${ }^{14}$ Cf. M. Praz, La Chair, la mort et le diable dans la littérature du XIXe siècle. Le Romantisme noir, trad. C. Thompson Pasquali, Paris, Gallimard, 1999, p. 160-162. II ne faut pas négliger l'importance de la littérature du XVIII siècle influencée par la " mode orientale ", dont les Lettres persanes qui bien souvent " donnent l'impression d'un chapitre des Mille et une Nuits habillé à la mode par un philosophe libertin " (P. Martino, L'Orient dans la littérature française au XVIIe et au XVIIIe siècle, Genève, Slatkine Reprints, 1970, p. 295 et le volume tout entier pour l'image de l'Orient dans la littérature française aux XVIIe-XVIIIIe siècles).

${ }^{15}$ Cf. V. Hugo, "Clair de lune ", [dans :] CEuvres complètes : Odes et Ballades, Essais et Poésie diverses. Les Orientales, Paris, Ollendorf, 1912, p. 670.

${ }^{16} \mathrm{Cf}$. V. Hugo, "La Sultane favorite ", [dans :] CEuvres complètes : Odes et Ballades, Essais et Poésie diverses. Les Orientales, op. cit., p. 673. 
éblouissante ; I'usage fréquent du bain a rendu leur peau très lisse et très belle. [...] J'ai eu un plaisir singulier à voir cette cérémonie. ${ }^{17}$

La description a d'ailleurs servi d'inspiration à Ingres pour son fameux Bain turc ${ }^{18}$.

En effet, la sensualité orientale, décrite déjà abondamment au XVIIIe siècle, battra son plein au siècle suivant. Pourquoi ? Parce que c'est une époque hypocrite quant à sa sexualité. Alain Corbin ne l'a-t-il pas nommée "le temps des oies blanches et des bordels "? "Voilà un siècle bien mal dans sa peau !", explique I'historien, "Siècle hypocrite qui réprime le sexe mais en est obsédé. Traque la nudité mais regarde par les trous de serrure. Corsète le couple conjugal mais promeut les bordels ${ }^{19}$. La femme orientale lascive, habile dans l'art de l'amour, devient un fantasme privilégié de ceux qui ne voient jamais leurs femmes entièrement dénudées. C'est la raison pour laquelle les odalisques peuplent les tableaux et poèmes de l'époque. L'hôtesse arabe du poème hugolien, mis en musique par Georges Bizet, ne promet-elle pas au " beau jeune homme blanc, bel oiseau passager " "le jeune sein de [s]es sœurs ", leur danse sensuelle, l'obéissance et la soumission ${ }^{20}$ ? Au XIXe siècle, l'Orient permet de visualiser ses fantasmes, de verbaliser ses désirs.

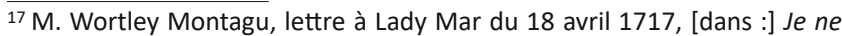
mens pas autant que les autres voyageurs. Lettres choisies, 1716-1718, trad. P. H. Anson, Paris, Payot, 2012, p. 143-144.

${ }^{18} \mathrm{Cf}$. L. Thornton, La femme dans la peinture orientaliste, trad. J. Coignard, Y. Thoraval, Tours, ACR PocheCouleur, 1993, p. 72. Pour d'autres relations de voyage qui ont bien influencé la perception de l'Orient au XVIII et au XIXe siècles et particulièrement ses représentations iconographiques, voir I. Apostolou, L'orientalisme des voyageurs français au XVIIle siècle. Une iconographie de l'Orient méditerranéen, Paris, PUPS, 2009, p. 260 et suivantes.

${ }^{19} \mathrm{~A}$. Corbin, " C'est le temps des oies blanches et des bordels. Propos recueillis par D. Simonnet ", [dans :] L'Express, 1er août 2002, https:// www.lexpress.fr/informations/alain-corbin-c-est-le-temps-des-oies-blanches -et-des-bordels_648919.html.

20 V. Hugo, "Adieux de l'hôtesse arabe ", [dans :] CEuvres complètes : Odes et Ballades, Essais et Poésie diverses. Les Orientales, op. cit., p. 701 


\section{La rencontre : les écrivains partent en Orient}

Victor Hugo n'a jamais mis le pied en Orient. Sa vision du Levant, servant d'inspiration à tant de jeunes (Gustave Flaubert y compris ${ }^{21}$ ), n'a jamais été confrontée à la réalité. Et pourtant le XIXe siècle est celui des voyages. Byron et Chateaubriand en ont indiqué la direction : le Levant. Ceux qui partent en Orient retrouvent-ils une contrée tissée de rêves, peintures et textes de l'époque ? "Pour que ce nom [Orient] produise à l'esprit de quelqu'un son plein et entier effet, il faut, sur toute chose, n'avoir jamais été dans la contrée mal déterminée qu'il désigne ", répond Paul Valéry ${ }^{22}$. On se rappelle la lettre ouverte où Gérard de Nerval-voyageur conseillait à Théophile Gautier de ne pas aller en Égypte pour ne pas perdre l'image "véritable " de l'Orient : "Oh ! reste à Paris, et puisse le succès de ton ballet se prolonger jusqu'à mon retour ! Je retrouverai à l'Opéra le Caire véritable, l’Égypte immaculée, l'Orient qui m'échappe... ${ }^{23}$.

Même si Nerval semble déçu de ne pas avoir retrouvé en Orient la contrée des Mille et une nuits, ce n'est pas la déception qui nourrit son Voyage en Orient. Les relations de voyage se conforment d'habitude, d'une manière ou d'une autre, à l'image idéalisée de l'Orient ${ }^{24}$. De là, des

\footnotetext{
21 Pour l'influence des romantiques (et avant tout leur image de l'Orient) sur Flaubert, cf. R. Giraud, The Unheroic Hero in the Novels of Stendhal, Balzac and Flaubert, New Brunswick, Rutgers University Press, 1957, s. 157-159 ou L. Czyba, "Le Voyage en Orient de Gustave Flaubert ", [dans :] Acta Universitatis Lodziensis. Folia Literaria, 1994, n 35, p. 71. Sarga Moussa parle même de l'importance d'un poème précis, déjà cité, "Adieux de l'hôtesse arabe " (Cf. S. Moussa, " Flaubert ou l'Orient à corps perdu ", [dans :] Revue des Lettres et de Traduction, 1999, n 5, p. 193194).

22 P. Valéry, "Préface ", [dans :] R. Bezombes, L'Exotisme dans l'art et la pensée, 1953, cité d'après J.-M. Moura, Lire l'exotisme, Paris, Dunod, 1992, p. III.

${ }^{23}$ G. de Nerval, " À mon ami Théophile Gautier », [dans :] CEuvres complètes. I, J. Guillaume, C. Pichois (éd. critique), Paris, Gallimard, 1989, p. 766.

${ }^{24}$ D’ailleurs, pour que l'image transférée soit acceptée par le public, il faut,
} 
paradoxes qui font parfois sourire. Eugène Fromentin, peintre et écrivain, se rend trois fois en Algérie, à peu près à l'époque du périple flaubertien, et publie ensuite deux récits de voyage : Un été dans le Sahara et Une année dans le Sahel. II est curieux de voir comment Fromentin décrit la chaleur si difficile à supporter dans cette partie du monde. Le plus souvent, il semble vouloir qu'il fasse encore plus chaud. En outre, on a l'impression qu'il supporte la chaleur mieux que les indigènes ${ }^{25}$. "Mon corps, comme mon âme, est fils du soleil, il lui faut la lumière ; il lui faut ce rayon de vie que cet astre darde, non pas du sein déchiré de nos nuages d'Occident, mais du fond de ce ciel de pourpre qui ressemble à la gueule de la fournaise ", déclarait Lamartine ${ }^{26}$. Peut-on dire que le soleil fatigue face à un tel modèle 27 ?

Et Flaubert ? II est sûrement excité par son voyage. "Impression solennelle et inquiète quand j'ai senti mon pied s'appuyer sur la terre d'Égypte ", note-t-il le 15 novembre 1849, en débarquant à Alexandrie (VO, 77). La déception apparaît-elle ? Sans doute. N'écrit-il pas à Louis Bouilhet :

Ô Orient, où es-tu ? - ॥ ne sera bientôt plus que dans le soleil. À Constantinople, la plupart des hommes sont habillés à l'européenne [...]

en quelque sorte, se soumettre à ce qui a déjà été dit. C'est la poétique même du récit de voyage, forme toujours répétitive. Cf. J.-P. Moura, Lire l'exotisme, op. cit., p. 73. Edward Saïd en parle de façon brutale : «Écrire sur l'Orient [...], c'est soit faire paraître une démystification bouleversante des images recueillies dans des textes, soit se confier dans l'Orient dont parlait Hugo [...] ». E. Saïd, L'Orientalisme. L'Orient créé par l'Occident, op. cit., p. 120.

${ }^{25}$ Cf. E. Fromentin, Un été dans le Sahara, [dans :] CEuvres complètes, G. Sagnes (éd. critique), Paris, Gallimard, 1984, p. 35-39 et passim. Né en 1820 et parti en Afrique du Nord en 1846 et puis en 1847, Fromentin est un voyageur appartenant à la génération de Flaubert. Il est intéressant de voir comment leur sensibilité différente influence leurs notes de voyage.

${ }^{26}$ A. de Lamartine, Voyage en Orient, Paris, Hachette, 1913, t. 1, p. 20.

27 Le même Fromentin meurt presque de fatigue et de chaleur (et le déclare) lorsqu'il voyage en Égypte à l'occasion de l'inauguration du canal de Suez, une vingtaine d'années plus tard ( $C f$. E. Fromentin, Carnets du voyage en Égypte, [dans :] CEuvres complètes, op. cit., p. 1049-1116). 
Dans cent ans d'ici, le harem, envahi graduellement par la fréquentation des dames franques, coulera de soi tout seul, sous le feuilleton et le vaudeville. Bientôt, le voile, déjà de plus en plus mince, s'en ira de la figure des femmes, et le musulmanisme avec lui s'envolera tout à fait. ${ }^{28}$

Flaubert cherche de l'exotisme, l'Orient qu'il connaît de ses lectures et de ses rêves ${ }^{29}$. Parfois, il en trouve. C'est pourquoi dans l'une de ses lettres, il affirme que son périple est partiellement " une trouvaille " et partiellement " une retrouvaille ${ }^{30}$. Parfois, il en devient las ${ }^{31}$. Et pourtant, il est généralement admis que son expérience de voyage a été réussie et bien importante pour son œuvre postérieure 32 .

\section{La sexualisation de l'Orient : un désir assouvi}

Si Flaubert n'est pas déçu de son voyage, c'est partiellement à cause de l'Orient fort sexualisé qu'il retrouve lors de son périple. II en parle dans ses notes et lettres, spécialement celles envoyées à Louis Bouilhet ${ }^{33}$. Appelé par

$28 \mathrm{G}$. Flaubert, lettre à Louis Bouilhet du 19 décembre 1850, [dans :] Correspondance. I (janvier 1830 à juin 1851), J. Bruneau (éd. critique), Paris, Gallimard, 1973, p. 730.

${ }^{29}$ Cf. Ph. Desan, "L'Autorité Orientaliste de Flaubert ", [dans :] Nottingham French Studies, 1983, n 22 (1), p. 15-24 ou S. Moussa, « Flaubert ou l'Orient à corps perdu », op. cit., p. 204-205.

${ }^{30} \mathrm{Cf}$. G. Flaubert, lettre à Louis Bouilhet du 1er décembre 1849, [dans :] Correspondance. I, op. cit., p. 538.

31 Maxime Du Camp l'accusait de ne pas être satisfait de leur voyage : " les temples lui paraissaient toujours les mêmes, les paysages toujours semblables, les mosquées toujours pareilles » (M. Du Camp, Souvenirs littéraires, Paris, Hachette, 1906, t. 1, p. 351-352). Flaubert le confirme dans ses notes : " les temples égyptiens m’embêtent profondément » (G. Flaubert, Voyage en Orient, op. cit., p. 158).

${ }^{32}$ Cf. par exemple L. Czyba, "Le Voyage en Orient de Gustave Flaubert ", op. cit., p. 76. À en croire Claudine Gothot-Mersch, l'écrivain n'a pas publié une relation de voyage uniquement parce qu'il la considérait comme " un genre mineur » (C. Gothot-Mersch, "Préface ", [dans :] G. Flaubert, Voyage en Orient, op. cit., p. 28).

33 Pour une signification intéressante de ses lettres, qui semblent s'inscrire dans la mode littéraire de l'époque, voir A. Buisine, L'Orient voilé, op. cit., p. 111. 
Dennis Porter " voyageur pervers ${ }^{34}$, il se plaît à décrire des situations salaces : un spectacle de saltimbanques où un médecin n'ouvre la porte qu'à des prostituées et sodomise son âne $(V O, 84-85)$; un garçon, ou un nain, qui propose à l'écrivain de lui apporter " [s]a mère à baiser " et lui souhaite " toutes sortes de prospérités, surtout d'avoir un très long vit " $(\mathrm{VO}, 85)$. Flaubert rapporte aussi I'histoire du bouffon de Méhémet Ali qui " prit une femme dans un bazar et la foutit sur le devant de la boutique coram populo ", celle d'un enfant enculé par un singe, ou celle d'un marabout mort " épuisé par la masturbation de toutes les femmes qui allaient le visiter " $(V O, 89)$. ॥ semble tout à fait excité quand, lors de sa visite à l'hôpital civil de l'Esbekieh, " une vieille femme [I]e priait de la baiser [et] exhibait son flasque et long téton pendant jusqu'au nombril et tapait dessus " $(V O, 106)$. Dans une lettre à Louis Bouilhet, il raconte comment un masseur s'est mis à le masturber dans un bain public ${ }^{35}$. Les descriptions, plus ou moins détaillées, de nombreux actes sexuels avec des danseuses égyptiennes et prostituées, dont la fameuse Kuchiuk Hanem, sont généralement connues ${ }^{36}$.

34 Cf. D. Porter, Haunted Journeys. Desire and Transgression in European Travel Writing, Princeton, Princeton University Press, 1991, p. 164. Porter souligne aussi que les moments les plus mémorables du voyage flaubertien relèvent de l'érotisme et de la pornographie. Cf. Ibidem, p. 175-176. 35 Cf. G. Flaubert, lettre à Louis Bouilhet du 15 janvier 1850, [dans :] Correspondance. I, op. cit., p. 572-573.

36 Par exemple Soirée chez la Triestine (G. Flaubert, Voyage en Orient, op. cit., p. 86-88), Un lupanar primitif (Ibidem, p. 123), Re-lupanar (Ibidem, p. 126), Kuchiuk-Hanem (Ibidem, p. 132-133), etc. L'analyse des expériences flaubertiennes avec les prostituées en tant que rencontre de l'idéal (du rêve) et du réel est présentée de façon très convaincante par A. Tooke, "Flaubert : Views of the Orient ", [dans :] M. Topping (dir.), Eastern Voyages, Western Visions. French Writing and Painting of the Orient, Bern, Peter Lang, 2004, p. 181-183. Pour le caractère très réaliste des descriptions salaces qui deviennent parfois exagérées et caricaturales, voir A. Buisine, L'Orient voilé, op. cit., p. 111. Pour leur " théâtralité ", A. Buisine, L'Orient voilé, op. cit., p. 111 et S. Moussa, "Flaubert ou l'Orient à corps perdu », op. cit., p. 209. 
Selon Alain Buisine, "le véritable désir de l'Orient tel qu'il ne cesse d'obséder tout l'Occident [...] est le désir de voile " alors que Flaubert passe son temps à tout dévoiler. Il désire que l'Orient " se dévoile " à lui dans toute sa nudité, y compris sexuelle ${ }^{37}$. Jean Potocki, dans ses Lettres de Turquie et d'Égypte (publiées en 1788), parle des danseuses décolletées, "prêtresses de la volupté "38. "Je ne pense pas que la dépravation ait jamais été portée nulle part à un degré aussi honteux ${ }^{39}$, déclare Auguste de Forbin, futur directeur général du Louvre, en décrivant le Caire du début du XIXe siècle. L'image de l'Orient lascif où tout est permis et aucune règle morale n'est observée est déjà bien connue, mais elle a toujours été suggérée plutôt que décrite ; pour suivre la belle métaphore d'Alain Buisine, elle est restée "voilée ». Flaubert, pourtant, pour qui le voyage devient " l'exaltation des sens et l'occasion de découvrir des expériences érotiques très fortes $\aleph^{40}$, n'hésite pas à décrire tout ce qu'il voit et éprouve dans un langage bien cru41. C'est du nouveau. Dans son carnet de voyage, qui date à peu près de la même époque (1848), Eugène Fromentin parle des "femmes presque nues "

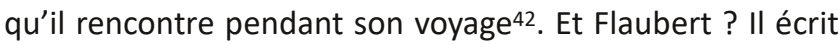

37 A. Buisine, L'Orient voilé, op. cit., p. 107-111. Pourtant, l'intérêt de Flaubert pour la sexualité et l'obscénité dépasse le cadre de son voyage, cf. F. Brown, "Flaubert in Egypt ", [dans :] New England Review, 2004, n० 4 (25), p. 42-43 ou B. Vinken, "Le continent noir du désir masculin : Colet et Flaubert, encore ", [dans :] Flaubert [En ligne], 2010, n 3, http:// journals.openedition.org/flaubert/968.

38 J. Potocki, "Lettre XIV ", [dans :] Voyages en Turquie et en Égypte, en Hollande, au Maroc, D. Beauvois (éd. critique), Paris, Fayard, 1980, p. 88.

${ }^{39}$ A. de Forbin, "Voyage dans le Levant ", [dans :] Le voyage en Orient. Anthologie des voyageurs français dans le Levant au XIXe siècle, op. cit., p. 841.

40 Th. Poyet, "Le voyeur et sa sexualité. Flaubert ou l'interdit des sens levé pour rien », op. cit., p. 169.

${ }^{41}$ Cf. D. Porter, Haunted Journeys. Desire and Transgression in European Travel Writing, op. cit., p. 177.

42 E. Fromentin, Carnets de voyage, [dans :] CEuvres complètes, op. cit., p. 941. 
à son frère Achille de manière enfantine : « Ah ! j'en ai t'y vu de ces tétons! j'en ai t'y vu ! j'en ai t'y vu ! »43.

L'écrivain veut aussi profiter de ce qu'il voit : il cherche des maisons closes clandestines, achète des mèches de cheveux de femmes arabes, que leurs maris coupent impitoyablement à leurs épouses en pleurs moyennant quelques piastres $(\mathrm{VO}, 166)$. Bref, il se comporte parfois en dominateur européen, ce qui lui sera tellement reproché par Edward Saïd44. Force est de constater que cette attitude n'est pourtant pas tellement nouvelle. Dans les années 1830 , le prince allemand Hermann von PücklerMuskau s'achète au marché d'esclaves du Caire une adolescente abyssinienne qu'il nomme "Mahbuba ", la BienAimée, et qu'il fait venir en Europe (la jeune fille est morte peu après $)^{45}$. On pense tout de suite à la Zeynab nervalienne qui est, certes, une création littéraire, mais on ne peut pas oublier la lettre de Nerval à Gautier :

Le Fonfride est assez convenable. II a acheté une esclave indienne et comme il voulait me la faire baiser je n'ai pas voulu, alors il ne l'a pas baisée non plus, nous en sommes là. Cette femme nous coûte cher et nous ne savons plus guère qu'en faire. On a d'autres femmes tant qu'on veut ${ }^{46}$.

Ce discours peu typique pour le poète délicat montre néanmoins une sorte d'auto-censure ou d'auto-contrôle jamais ressentis chez Flaubert. La visite chez Kuchiuk-

43 G. Flaubert, lettre au frère du 15 décembre 1949, [dans :] Correspondance. I, op. cit., p. 555.

44 E. Saïd, L'Orientalisme. L'Orient créé par l'Occident, op. cit., p. 18 et l'article plutôt critique envers l'attitude de Flaubert par Lisa Lowe : L. Lowe, "The Orient as Woman in Flaubert's Salammbô and Voyage en Orient ", [dans :] Comparative Literature Studies, 1986, n 1 (23), p. 4458.

45 E. Kleßmann, Fürst Pückler und Machbuba, Berlin, Rowohlt Verlag, 1998. Dans une lettre à sa mère, le poète romantique polonais Juliusz Słowacki regrette de ne pas avoir acheté une esclave abyssinienne au Caire. Cf. J. Słowacki, list z 3 października 1837 r., [dans :] Listy do matki, Z. Krzyżanowska (éd. critique), Kraków, Wydawnictwo Ossolińskich, 1979, p. 276.

${ }^{46} \mathrm{G}$. de Nerval, lettre à Théophile Gautier du 2 mai 1843, [dans :], CEuvres complètes, op. cit., p. 1396. 
Hanem, la célèbre almée, qui vient de sortir des bains est un stimulus tellement fort que les deux voyageurs, Flaubert et Du Camp, ne sont pas capables de retenir leur désir : "Elle nous a demandé si nous voulions nous amuser. Maxime a d'abord demandé de s'amuser seul avec elle et est descendu dans une salle du rez-de-chaussée [...] après M. Du Camp ç'a été M. Flaubert » $(V O, 133)$.

Chose intéressante, Du Camp décrit la même scène dans sa relation de voyage : "Après tous les souhaits de bienvenue impérieusement exigés par la politesse arabe, Koutchouk-Hanem me prit la main et me conduisit dans une grande chambre carrée ${ }^{47}$, pour y danser, bien évidemment! Du Camp a publié sa relation, Flaubert n'avait pas un tel projet. La différence montre bien pourtant le double caractère du voyage oriental de l'époque : ce qui se faisait (discours de Flaubert) et ce qui se publiait (discours de Maxime Du Camp).

\section{Conclusion}

Le voyage flaubertien en Orient, et particulièrement sa partie égyptienne, qui se reflète dans ses notes et lettres de voyage, montre-il donc une révolution ou une évolution dans l'approche de l'Orient ? Les deux, sans doute. Une révolution discursive : Flaubert est le premier à opposer à la vision poétiquement sensuelle de l'Orient les descriptions naturalistes des organes sexuels ${ }^{48}$. II adopte " une position volontairement "immorale", antihumaniste ${ }^{49}$ et en parle ouvertement, " médicalement, brutalement, indécemment ${ }^{50}$. C'est le même réalisme

\footnotetext{
47 M. Du Camp, Le Nil : Égypte et Nubie, Paris, A. Bourdilliat, 1860, p. 116. Pour d'autres exemples où la description de Flaubert varie de façon significative de celle de Maxime du Camp, voir A. Buisine, L'Orient voilé, op. cit., p. 105-106.

48 Selon Claudine Gothot-Mersch, c'était " osé pour l'époque 》. Cf. C. Gothot-Mersch, "Préface », op. cit., p. 27.

${ }^{49} \mathrm{~S}$. Moussa, "Flaubert ou l'Orient à corps perdu », op. cit., p. 208.

${ }^{50} \mathrm{~A}$. Buisine, L'Orient voilé, op. cit., p. 105.
} 
cru que celui des Femmes d'Alger de Delacroix. Même si le texte n'était pas destiné à être publié, il diffère d'autres carnets de voyage de l'époque et même d'autres lettres ${ }^{51}$. Certes, Flaubert se censurait rarement dans ses écrits privés, mais utiliser une langue très biologique, voire vulgaire, pour parler de la belle odalisque, rêve de l'époque, peut être traité de révolutionnaire. Et l'aspect évolutionnaire ? II réside dans toutes ces expériences sexuelles décrites par Flaubert. Quand on entend parler d'un fruit délicieux qui pousse dans un endroit lointain, n'a-t-on pas envie d'y goûter une fois sur place ? Flaubert a grandi emmailloté dans les rêves sensuels de l'Orient, des houris à la peau olivâtre et aux yeux noircis de khôl52. Comme bien des autres, il a voulu réaliser ces rêves et il l'a fait, grâce aux danseuses et prostituées. C'est un pas vers le tourisme sexuel qui battra son plein quelques décennies après.

En parlant de l'Orient du XIXe siècle, Jean-Marie Carré l'appelait " facile et décoratif, fait de bric-à-brac exotique, d'illuminations aveuglantes, d'éclats brusques et contrastés, d'anecdotes et de détail ${ }^{53}$. Dans ce mélange de poèmes, peintures ou relations de voyage qui constitue ce qu'on nomme aujourd'hui l'orientalisme du XIXe siècle, le voyage de Flaubert - ainsi que le tableau de Delacroix -, est visiblement différent, car il apporte un malaise face au rêve, une gêne face à la convention ; au demeurant, il marque un changement (r)évolutionnaire.

Date de réception de l'article : 30.12.2017. Date d'acceptation de l'article : 20.05.2018.

51 Cf. F. Lacoste, "L'Orient de Flaubert », op. cit., p. 78. Dennis Porter appelle même le Voyage en Orient " a disturbing and symptomatic text " (un texte dérangeant et symptomatique). D Porter, Haunted Journeys. Desire and Transgression in European Travel Writing, op. cit., p. 169.

52 Cf. ses œuvres de jeunesse, par exemple Rage et impuissance ou Novembre.

53 J.-M. Carré, "Introduction ", [dans :] E. Fromentin, Voyage en Égypte, J.-M. Carré (éd. critique), Paris, Éditions Montaigne, 1935, p. 20. 


\section{bibliographie}

Apostolou I., L'orientalisme des voyageurs français au XVIIIe siècle. Une iconographie de l'Orient méditerranéen, Paris, PUPS, 2009.

Berchet J.-C., "Introduction ", [dans :] Le voyage en Orient. Anthologie des voyageurs français dans le Levant au XIXe siècle, J.-C. Berchet (éd. critique), Paris, Robert Laffont, 1985.

Brown F., "Flaubert in Egypt », [dans :] New England Review, 2004, n 4 (25). Buisine A., L'Orient voilé, Paris, Zulma, 1993.

Carré J.-M., "Introduction ", [dans :] E. Fromentin, Voyage en Égypte, J.-M. Carré (éd. critique), Paris, Éditions Montaigne, 1935.

Cogitore C., "Femmes d'Alger dans leur appartement ", 1834, Eugène Delacroix, ARTE France, 2015 (9e épisode de la série Les petits secrets des grands tableaux).

Corbin A., " C'est le temps des oies blanches et des bordels. Propos recueillis par D. Simonnet ", [dans :] L'Express, $1^{\text {er }}$ août 2002, https:// www.lexpress.fr/informations/alain-corbin-c-est-le-temps-des-oies-blancheset-des-bordels_648919.html.

Czyba L., "Le Voyage en Orient de Gustave Flaubert ", [dans :] Acta Universitatis Lodziensis. Folia Literaria, 1994, n 35.

Desan Ph., "L'Autorité Orientaliste de Flaubert ", [dans :] Nottingham French Studies, 1983, n० 22 (1).

Du Camp M., Le Nil : Égypte et Nubie, Paris, A. Bourdilliat, 1860.

Flaubert G., Correspondance. I (janvier 1830 à juin 1851), J. Bruneau (éd. critique), Paris, Gallimard, 1973.

Flaubert G., Correspondance. II (juillet 1851 - décembre 1858), J. Bruneau (éd. critique), Paris, Gallimard, 1980.

Flaubert G., Rage et impuissance, [dans :] Idem, Mémoires d'un fou, Novembre et autres textes de jeunesse, Y. Leclerc (éd. critique), Paris, Flammarion, 1991.

Flaubert G., Voyage en Orient, C. Gothot-Mersch (éd. critique), Paris, Gallimard, 2006.

Forbin A. de, Voyage dans le Levant, [dans :] Le voyage en Orient. Anthologie des voyageurs français dans le Levant au XIXe siècle, J.-C. Berchet (éd. critique), Paris, Robert Laffont, 1985.

Fromentin E., Carnets de voyage, [dans :] CEuvres complètes, G. Sagnes (éd. critique), Paris, Gallimard, 1984.

Fromentin E., Carnets du voyage en Égypte, [dans :] CEuvres complètes, G. Sagnes (éd. critique), Paris, Gallimard, 1984.

Fromentin E., Un été dans le Sahara, [dans :] CEuvres complètes, G. Sagnes (éd. critique), Paris, Gallimard, 1984.

Genet-Delacroix M.-C., "Delacroix et les "Néos" : pour le vrai contre le faux ", [dans :] Sociétés \& Représentations, 2005, n 20.

Giraud R., The Unheroic Hero in the Novels of Stendhal, Balzac and Flaubert, New Brunswick, Rutgers University Press, 1957.

Gothot-Mersch C., "Préface ", [dans :] G. Flaubert, Voyage en Orient, C. Gothot-Mersch (éd. critique), Paris, Gallimard, 2006.

Hugo V., CEuvres complètes : Odes et Ballades, Essais et Poésie diverses. Les Orientales, Paris, Ollendorf, 1912. 
Kleßmann E., Fürst Pückler und Machbuba, Berlin, Rowohlt Verlag, 1998. Lacoste F., "L'Orient de Flaubert », [dans :] Romantisme, 2003, n 119. Lamartine A. de, Voyage en Orient, Paris, Hachette, 1913, t. 1.

Larousse P., Grand dictionnaire universel du XIXe siècle, Paris, Larousse, 1874, t. 11.

Lowe L., "The Orient as Woman in Flaubert's Salammbô and Voyage en Orient ", [dans :] Comparative Literature Studies, 1986, n 1 (23).

Martino P., L'Orient dans la littérature française au XVIIe et au XVIIIe siècle, Genève, Slatkine Reprints, 1970.

Moura J.-M., Lire l'exotisme, Paris, Dunod, 1992.

Moussa S., "Signatures : ombre et lumière de l'écrivain dans la Correspondance d'Orient de Flaubert », [dans :] Littérature, 1996, n 104.

Moussa S., "Flaubert ou l'Orient à corps perdu », [dans :] Revue des Lettres et de Traduction, 1999, nº 5 .

Nerval G. de, Le Voyage en Orient, M. Jeanneret (éd. critique), Paris, Garnier-Flammarion, 1980, t. 1.

Nerval G. de, CEuvres complètes. I, J. Guillaume, C. Pichois (éd. critique), Paris, Gallimard, 1989.

Porter D., Haunted Journeys. Desire and Transgression in European Travel Writing, Princeton, Princeton University Press, 1991.

Potocki J., Voyages en Turquie et en Égypte, en Hollande, au Maroc, D. Beauvois (éd. critique), Paris, Fayard, 1980.

Poyet Th., " Le voyeur et sa sexualité. Flaubert ou l'interdit des sens levé pour rien ", [dans :] Ph. Antoine (dir.), Sur les pas de Flaubert. Approches sensibles du paysage, Amsterdam, Rodopi, 2014.

Praz M., La Chair, la mort et le diable dans la littérature du XIXe siècle. Le Romantisme noir, trad. C. Thompson Pasquali, Paris, Gallimard, 1999.

Saïd E., L'Orientalisme. L'Orient créé par l'Occident, trad. C. Malamoud, Paris, Éditions du Seuil, 2005.

Słowacki J., Listy do matki, Z. Krzyżanowska (éd. critique), Kraków, Wydawnictwo Ossolińskich, 1979.

Sturm E., " Voyage en Égypte by Gustave Flaubert and Pierre-Marc de Biasi ", [dans :] The French Review, 1993, n 2 (67).

Thornton L., La femme dans la peinture orientaliste, trad. J. Coignard, Y. Thoraval, Tours, ACR PocheCouleur, 1993.

Tooke A., "Flaubert : Views of the Orient ", [dans :] M. Topping (dir.), Eastern Voyages, Western Visions. French Writing and Painting of the Orient, Bern, Peter Lang, 2004.

Vauday P., La Décolonisation du tableau. Art et poétique au XIXe siècle. Delacroix, Gauguin, Paris, Seuil, 2006.

Vinken B., " Le continent noir du désir masculin : Colet et Flaubert, encore ", [dans :] Flaubert [En ligne], 2010, n 3, http://journals.open edition.org/flaubert/968.

Wortley Montagu M., Je ne mens pas autant que les autres voyageurs. Lettres choisies, 1716-1718, trad. P. H. Anson, Paris, Payot, 2012. 


\section{abstract}

The Oriental Travelogue by Gustave Flaubert: a Revolution or an Evolution in the Approach toward the Middle East

The aim of the present paper is to answer the question whether the Oriental travelogue by Gustave Flaubert, notorious for the bawdy descriptions of various sexual experience the author gains during his trip, represents a revolution or an evolution in the literary approach toward the Middle East. The paper is divided into three parts. The first describes briefly the history of the Oriental dream and the 19th-century predilection for the voluptuous odalisques. The second discusses a few Oriental travelogues and the meeting of the dream with the reality while the last one analyses the sexualisation of the Oriental experience becoming increasingly important during the 19th century.

\section{keywords}

Flaubert, travel literature, Oriental travelogue, revolution, evolution

\section{mots-clés}

Flaubert, littérature de voyage, voyage en Orient, révolution, évolution

\section{malgorzata soko lowicz}

Małgorzata Sokołowicz, maître de conférences à l'Institut d'études romanes de l'Université de Varsovie et à l'Université de musique FrédéricChopin, est l'auteur du livre La Catégorie du héros romantique dans la poésie française et polonaise au XIXe siècle (2014) et de nombreux articles sur les relations entre littérature et art, l'orientalisme et les questions identitaires. 